\title{
Neutrophil: Lymphocyte Ratio Predicts Coronary Artery Disease Severity in Non-ST Elevation Myocardial Infarction Using Syntax Score
}

\author{
Ahmed Elamragy ${ }^{\text {a }}$, Ahmed Hussein ${ }^{\text {b }}$, Ayman Kaddah ${ }^{\text {a }}$, Marwa Meshaal ${ }^{\text {a }}$
}

a Department of cardiology,
faculty of medicine, Cairo
University, Cairo, Egypt.
b Department of cardiology,
Military Production Hospital,
Cairo, Egypt.

Correspondence to: Ahmed

Elamragy, Department of cardiology, faculty of medicine, Cairo University, Cairo, Egypt

\section{Email:}

ahmed.elamragy@kasralainy.edu.eg

Received: 5 December 2020

Accepted: 29 December 2020

\begin{abstract}
:
Background: Inflammation plays a pivotal role in the initiation and progression of atherosclerosis. Neutrophil:lymphocyte ratio (NLR) is a prognostic marker for several cardiovascular diseases, including non-ST-elevation myocardial infarction (NSTEMI). Syntax score (Sx score) is an anatomical score system for coronary artery disease (CAD) severity and can predict short- and long-term mortality in CAD intervention. Aim: To investigate the association between NLR and Sx score as a measure of CAD severity in patients with NSTEMI. Methods: A prospective study was performed over 12 months on patients presenting with NSTEMI.
\end{abstract} Complete blood count (for NLR calculation), s. urea, s. creatinine, s. lipid profile, and s. cardiac enzymes were withdrawn upon admission. Coronary angiography with Sx score calculation was done during the index hospitalization. Results: We studied 111 consecutive patients, 85 males $(76.6 \%)$ with a mean age of $55.3 \pm$ 9.8 years. In univariate analysis, a high $\mathrm{Sx}$ score $(\geq 33)$ was significantly associated with s. cholesterol, s. LDL, s. HDL, s. creatinine, s. CK, s. CK-MB, and NLR. In multivariate analysis, NLR was the only significant predictor of high Sx score [HR: 4.19 and 95\% CI: 1.7-10.5, p: 0.002]. In the ROC analysis, the NLR cutoff of $>7.1$ had $100 \%$ sensitivity and $94 \%$ specificity to predict a high Sx score (AUC 0.97, P<0.001). We found no similar data in the literature. Conclusion: NLR is significantly associated with CAD severity in patients with NSTEMI. Admission NLR > 7.1 predicts a high Sx score with 100\% sensitivity and 94\% specificity, thus helping in early and better risk stratification of NSTEMI.

Keywords: Inflammation; Neutrophil:lymphocyte ratio; Syntax; NSTEMI; Severity. 


\section{List of abbreviations}

ACS: acute coronary syndromes.

$\mathrm{CV}$ : cardiovascular.

NLR: Neutrophil: lymphocyte ratio.

CAD: Coronary artery disease.

STEMI: ST-elevation myocardial infarction.

NSTEMI: non-ST-elevation myocardial infarction.

Sx score: Syntax score.

eGFR: estimated glomerular filtration rate.

LDL: low-density lipoprotein.

HDL: high-density lipoprotein.

SD: standard deviation.

ROC: receiver operating characteristics curve.

AUC: area under the curve.

\section{Introduction}

Inflammation plays a major role in the initiation and progression of atherosclerosis and may lead to acute thrombotic complications $^{1}$. Increased levels of inflammatory markers are associated with the severity of coronary atherosclerosis and acute coronary syndromes (ACS) prognosis $^{2}$. Elevated leucocyte count was shown as a marker for cardiovascular (CV) risk prediction ${ }^{3}$.

However, more recent studies suggest that the neutrophil: lymphocyte ratio (NLR) may be more specific ${ }^{4}$-reflecting the proinflammatory state and therefore worse clinical outcomes in several $\mathrm{CV}$ diseases including coronary artery ectasia, stable coronary artery disease (CAD), ST-elevation myocardial infarction (STEMI), and nonST-elevation myocardial infarction (NSTEMI) ${ }^{5}$.

Although NSTEMI has lower in-hospital mortality than STEMI, the 6-month mortality is similar, and the 4-year mortality is even 2-fold higher. Risk stratification and management of NSTEMI in the acute phase could therefore be critical to the prevention of increased long-term mortality ${ }^{6}$.

There are several methods for assessing the severity of CAD in angiograms. Syntax score $(\mathrm{Sx}$ score) is an anatomical score system that quantifies coronary artery lesions in terms of their complexity, morphology, and location within the coronary vasculature. It may predict shortand long-term mortality after CAD intervention ${ }^{7}$.

The aim of this study was to investigate the association between the NLR and Sx scores as a measure of CAD severity and as a result, the outcome predictor following interventions in patients with NSTEMI. 


\section{Patients and Methods}

\section{Study population}

Over 12 months, this prospective study included 111 consecutive patients who presented with NSTEMI to two tertiary care hospitals and subsequently underwent coronary angiography during their index hospitalization. Diagnosis of NSTEMI was based on the guidelines of the American College of Cardiology ${ }^{8}$. Exclusion criteria included cardiogenic shock on admission, history of heart failure, acute/chronic inflammation or infection, chronic obstructive lung disease, malignancy, or chronic kidney disease (estimated glomerular filtration rate (eGFR) $<60$ $\mathrm{ml} / \mathrm{min} / 1.73 \mathrm{~m}^{2}$ ).

\section{Study methods}

All patients were screened for conventional CAD risk factors: smoking status, hypertension, diabetes mellitus, dyslipidemia. Hypertension was diagnosed if the blood pressure was $\geq 140 / 90 \mathrm{mmHg}$ and/or on treatment with antihypertensive medications ${ }^{9}$. Diabetes mellitus was diagnosed with fasting glucose $>126 \mathrm{mg} / \mathrm{dL}$ and/or treatment with hypoglycemic medications ${ }^{10}$. Dyslipidemia was diagnosed with fasting total cholesterol $>200 \mathrm{mg} / \mathrm{dL}$ or low-density lipoprotein (LDL) $\geq 130 \mathrm{mg} / \mathrm{dL}$ or when treated with a lipid-lowering drug ${ }^{11}$.

Twelve-leads resting electrocardiogram was performed upon admission to exclude persistent ST-segment elevation. Biochemical analysis was performed from the admission blood samples and the 12hour fasting blood samples collected on the following day. The complete blood count with differential count was analysed using Beckman-Coulter AU 2700 (Beckman Coulter Ireland Inc. Mervue, Galway, Ireland) while fasting blood glucose, serum urea, creatinine, CK, CK-MB, total cholesterol, triglycerides, LDL, and highdensity lipoprotein (HDL) were analysed using Automated Cobas c311. NLR was obtained from the differential leucocytic count, and eGFR was calculated to exclude chronic kidney disease.

During their index hospitalization, all patients underwent coronary angiography using the Judkins technique. The CAD severity analysis using the $\mathrm{Sx}$ score was carried out by 2 experienced interventional cardiologists unaware of the patients' clinic and laboratory results using a web-based calculator $^{12}$. Each coronary lesion with $a \geq$ $50 \%$ luminal obstruction in vessels $\geq 1.5$ $\mathrm{mm}$ was scored separately and added to provide the total Sx score ${ }^{13}$. 
All participants signed informed consents as per the study protocol approved by the Ethics Committee of Cairo University.

\section{Statistical Analysis}

Data analysis was performed using the SPSS 23.0 statistical package for Windows (SPSS Inc., Chicago, Ill, USA). Continuous data were presented as mean \pm standard deviation (SD).

Qualitative data were presented as numbers (percentages). Correlation analysis was done using Pearson's test for normally distributed data and Spearman's test for skewed data. Patients were divided into 2 groups based on the clinical significance of $\mathrm{Sx}$ score according to the SYNTAX trial ${ }^{14}$ : lowintermediate Sx group $(<33)$ and high $\mathrm{Sx}$ group ( $\geq 33)$.

Chi-square test was used to compare categorical variables while independent $\mathrm{t}$ tests were used for comparing continuous variables. Univariate logistic regression analysis was used to detect significant associations with high $\mathrm{Sx}$ which entered a multivariate analysis to detect the most significant independent variable.

Receiver operating characteristics curve (ROC) analysis was used to obtain the optimal cut-off values for predicting high $\mathrm{Sx}$ scores with significant sensitivity and specificity. The area under the curve (AUC) is an index of ROC accuracy: values close to 1.0 indicate high diagnostic accuracy. In all cases, a p-value $<0.05$ was regarded as statistically significant.

\section{Results}

\section{Baseline characteristics}

This study included 111 patients, 76.6\% were males. Other patients' characteristics are summarized in Table (1).

\section{Correlation Analysis}

Correlation analysis showed that $\mathrm{Sx}$ score was strongly and significantly correlated with NLR; while it had intermediately significant correlation with cardiac enzymes and LDL, and a weak correlation with age, HDL, and renal functions (Table 2).

\section{Sx score associations}

Univariate regression analysis showed that high Sx score was significantly associated with serum cholesterol, LDL, HDL, creatinine, CK, CK-MB levels, and NLR. In multivariate analysis, NLR was the only significant independent predictor of high $\mathrm{Sx}$ score [HR: 4.19 and 95\% CI: 1.7-10.5, p: 0.002]. (Table 3) 


\section{ROC Curve}

A ROC curve was plotted to detect the cut-

off point at which NLR detected a high Sx score. An NLR of $>7.1$ had $100 \%$ sensitivity and $94 \%$ specificity for detecting high Sx score: AUC 0.97, $p<0.001$ (Figure $1)$.

Table 1. Characteristics of the study population.

\begin{tabular}{ll}
\hline Variable & $\mathbf{N}(\%)$ \\
\hline Age (years) & $55.3 \pm 9.8^{\mathrm{a}}$ \\
Male gender & $85(76.6 \%)$ \\
Diabetes mellitus & $37(33.3 \%)$ \\
Hypertension & $60(54.1 \%)$ \\
Dyslipidemia & $72(64.9 \%)$ \\
Smoking & $44(39.6 \%)$ \\
NLR & $4.8 \pm 2$ \\
Syntax score & $14.6 \pm 9.7$ \\
\hline
\end{tabular}

NLR: neutrophil: lymphocyte ratio ${ }^{\text {a }}$ Mean \pm SD

Table 2. Correlation analysis between syntax score and different variables.

\begin{tabular}{|c|c|c|c|}
\hline & Variable & $\begin{array}{l}\text { Correlation } \\
\text { coefficient (r) }\end{array}$ & $p$-value \\
\hline 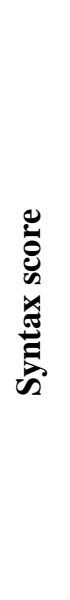 & $\begin{array}{l}\text { Age } \\
\text { Lipid profile } \\
\text { Cholesterol } \\
\text { HDL } \\
\text { HDL } \\
\text { Kidney function tests } \\
\quad \text { Urea } \\
\text { Creatinine } \\
\text { Cardiac biomarkers } \\
\text { CK } \\
\text { TLC CK-MB } \\
\text { NLR }\end{array}$ & $\begin{array}{l}0.18 \\
0.29^{\mathrm{a}} \\
0.51^{++} \\
0.47^{++} \\
0.18^{+} \\
0.73^{+}\end{array}$ & $\begin{array}{l}<0.0001^{\mathrm{c}} \\
<0.0001^{\mathrm{c}} \\
<0.0001^{\mathrm{c}} \\
0.02^{\mathrm{c}} \\
0.38 \\
0.054 \\
0.002^{\mathrm{c}} \\
<0.0001^{*} \\
<0.0001^{*} \\
0.06 \\
<0.0001^{*}\end{array}$ \\
\hline
\end{tabular}

LDL: low-density lipoprotein, HDL: high-density lipoprotein, CK: creatine kinase, CK-MB: creatine kinase M-band, TLC: total leucocytic count, NLR: neutrophil:Iymphocyte ratio.

${ }^{\text {a }}$ Pearson's correlation analysis.

'Spearman's correlation analysis.

${ }^{c} p$ values $<0.05$. 
Table 3. Multivariate regression analysis for high syntax score.

\begin{tabular}{llll}
\hline Variable & OR & $\mathbf{9 5 \%} \mathbf{C I}$ & $p$-value \\
\hline NLR & 4.19 & $1.7-10.5$ & $0.002^{\mathrm{a}}$ \\
Age & - & - & 0.359 \\
Creatinine & - & - & 0.055 \\
CK & - & - & 0.119 \\
CK-MB & - & - & 0.998 \\
Cholesterol & - & - & 0.243 \\
LDL & - & - & 0.180 \\
HDL & - & - & 0.320 \\
\hline
\end{tabular}

NLR: neutrophil: lymphocyte ratio, CK: creatine kinase, CK-MB: creatine kinase M-band, LDL: low-density lipoprotein, HDL: high-density lipoprotein.

${ }^{\mathrm{a}} p$-value $<0.05$.

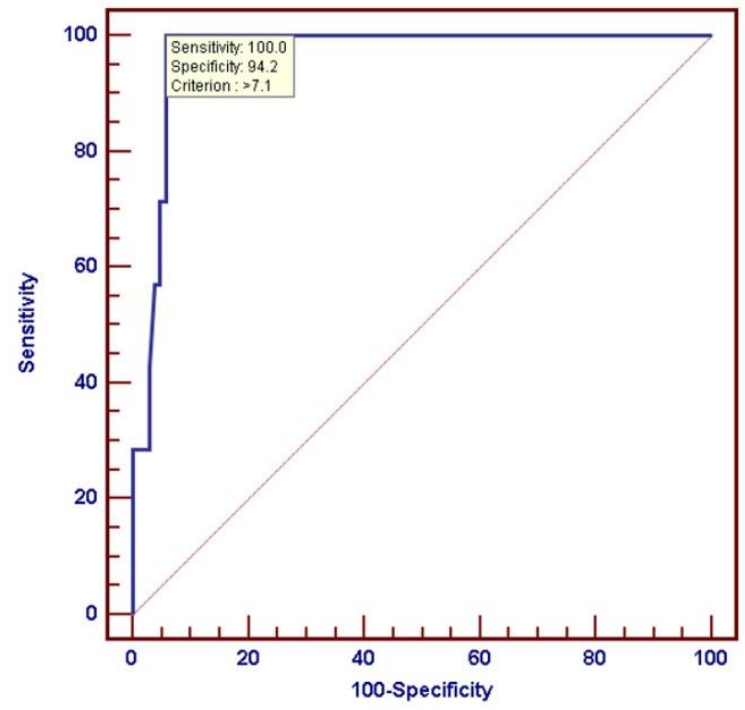

Figure 1. ROC curve of NLR for detection of high syntax score.

\section{Discussion}

The role of inflammation in the initiation and progression of coronary artery atherosclerosis is well established. There was an association between increased levels of inflammatory markers and the severity of
CAD and ACS prognosis. NLR is an indicator of baseline inflammatory response $^{15}$ and has been proposed as a prognostic marker of worse clinical outcomes in cardiovascular disease ${ }^{5}$. 
Although many studies have shown the NLR predictive value of CAD severity in patients with STEMI and stable CAD, its role in NSTEMI is less clear.

In this study, we found a strong significant correlation between NLR at the time of admission and $\mathrm{Sx}$ score. Its correlation was intermediate with cardiac enzymes and LDL cholesterol, and weak with age, HDL cholesterol, and renal functions. Multivariate analysis showed that NLR was the only independent predictor of high Sx scores. A previous study ${ }^{16}$ found similar results, but age was also significantly associated with CAD severity. However, the NLR association was strong and independent as well.

Other studies showed similar results in different clinical scenarios -STEMI and stable CAD. One study ${ }^{17}$ showed that NLR was significantly associated with CAD severity in patients with STEMI and that NLR was an independent predictor for $\mathrm{Sx}$ score. Another study ${ }^{18}$ showed that NLR was significantly associated with both the presence and severity of CAD in patients with stable CAD.

We found, through ROC analysis, that an NLR of $>7.1$ had a $100 \%$ sensitivity and a $94 \%$ specificity for the prediction of high $\mathrm{Sx}$ values (AUC 0.97, $\mathrm{p}<0.001$ ). To our knowledge, this has not been done or reported in the literature. This means that we could predict the CAD severity of NSTEMI patients with very high sensitivity and specificity simply by measuring their NLR at admission. This can improve the risk stratification and the early management planning of NSTEMI patients.

There were some limitations to this study. It was conducted on NSTEMI patients only. Therefore, the derived NLR cut-off point cannot be applied to all patients with acute coronary syndromes. The time from the onset of chest pain to hospital arrival was not measured. This may have affected the NLR levels of admission. Most of our study population were males. However, there was no selection bias as we enrolled consecutive patients without gender-based exclusions. Besides, gender was not an important predictor of the $\mathrm{Sx}$ score severity in the univariate analysis. Besides, the Sx score is a tool that assesses the complexity of coronary atherosclerosis and its impact on the clinical outcome following intervention; however, it is not a true measure of coronary atherosclerosis. But it is simple, user-friendly, and accessible. Finally, the new NLR cut-off requires prospective validation of its 
reproducibility for predicting CAD severity on admission.

\section{Conclusion}

NLR is significantly associated with severe CAD in NSTEMI. An NLR on admission > 7.1 could predict a syntax score of $>33$ with $100 \%$ sensitivity and $94 \%$ specificity: thus helping in early and better risk stratification of NSTEMI.

\section{References}

1. Papadimitraki ED, Boumpas DT. Inflammation and Atherosclerosis. In: Introduction to Translational Cardiovascular Research. Cham: Springer International Publishing; 2015: 217-238.

2. Kaya MG. Inflammation and coronary artery disease: as a new biomarker neutrophil/lymphocyte ratio. Turk Kardiyol Dern Arsivi-Archives Turkish Soc Cardiol. 2013; 41:191-192.

3. Lee CD, Folsom AR, Nieto FJ, Chambless LE, Shahar E, Wolfe DA. White blood cell count and incidence of coronary heart disease and ischemic stroke and mortality from cardiovascular disease in African-American and White men and women: atherosclerosis risk in communities study. Am $J$ Epidemiol. 2001; 154:758-64.

4. Horne BD, Anderson JL, John JM, Weaver A, Bair TL, Jensen KR, et al. Which White Blood Cell Subtypes Predict Increased Cardiovascular Risk? J Am Coll Cardiol. 2005; 45:1638-1643.

5. Chakrabarti AK, Gibson CM. The SYNTAX score: usefulness, limitations, and future directions. J Invasive Cardiol. 2011; 23:511-2.
6. Kaya MG, Sahin O, Akpek M, Duran M, Uysal OK, Karadavut S, et al. Relation Between Serum Total Bilirubin Levels and Severity of Coronary Artery Disease in Patients With Non-ST-Segment Elevation Myocardial Infarction. Angiology. 2014; 65:245-249.

7. van Gaal WJ, Ponnuthurai FA, Selvanayagam J, Testa L, Porto I, Neubauer S, et al. The Syntax score predicts peri-procedural myocardial necrosis during percutaneous coronary intervention. Int $J$ Cardiol. 2009; 135:60-65.

8. Amsterdam EA, Wenger NK, Brindis RG, Casey DE, Ganiats TG, Holmes DR, et al. 2014 AHA/ACC Guideline for the Management of Patients With Non-ST-Elevation Acute Coronary Syndromes: Executive Summary. Circulation. 2014; 130:2354-2394.

9. Chobanian A V., Bakris GL, Black HR, Cushman WC, Green LA, Izzo JL, et al. Joint National Committee on Prevention, Detection, Evaluation, and Treatment of High Blood Pressure. National Heart, Lung and BI, National High Blood Pressure Education Program Coordinating Committee. Seventh report of the Joint National Committee on Prevention, Detection, Evaluation, and Treatment of High Blood Pressure. Hypertens. 2003; 42:1206-52.

10. Professional Practice Committee for the Standards of Medical Care in Diabetes-2016. Diabetes Care. 2016; 39:S107-S108.

11. National Cholesterol Education Program (NCEP) Expert Panel on Detection, Evaluation and $\mathrm{T}$ of HBC in A (Adult TPI. Third Report of the National Cholesterol Education Program (NCEP) Expert Panel on Detection, Evaluation, and Treatment of High Blood Cholesterol in 
Adults (Adult Treatment Panel III) final report. Circulation. 2002; 106: 3143-421.

12. SYNTAX score working group. SYNTAX score calculator. http://www.syntaxscore.com/calculator.

13. Sianos G, Morel M-A, Kappetein AP, Morice M-C, Colombo A, Dawkins K, et al. The SYNTAX Score: an angiographic tool grading the complexity of coronary artery disease. EuroIntervention. 2005; 1:219-27.

14. Mohr FW, Morice M-C, Kappetein AP, Feldman TE, Ståhle E, Colombo A, et al. Coronary artery bypass graft surgery versus percutaneous coronary intervention in patients with three-vessel disease and left main coronary disease: 5-year follow-up of the randomised, clinical SYNTAX trial. Lancet. 2013 ; 381:62938.

15. Sarli B, Baktir AO, Saglam H, Arinc H, Kurtul S, Sivgin S, et al. Neutrophil-to-
Lymphocyte Ratio is Associated With Severity of Coronary Artery Ectasia. Angiology. 2014; 65:147-151.

16. Kurtul S, Sarli B, Baktir AO, Demirbas M, Saglam H, Doğan $\mathrm{Y}$, et al. Neutrophil to lymphocyte ratio predicts SYNTAX score in patients with non-ST segment elevation myocardial infarction. Int Heart J . 2015; 56:1821.

17. Sahin DY, Elbasan Z, Gür M, Yildiz A, Akpinar O, Icen $\mathrm{YK}$, et al. Neutrophil to lymphocyte ratio is associated with the severity of coronary artery disease in patients with STsegment elevation myocardial infarction. Angiology. 2013; 64:423-9.

18. Kaya A, Kurt M, Tanboga IH, Işık T, Günaydın ZY, Kaya Y, et al. Relation of neutrophil to lymphocyte ratio with the presence and severity of stable coronary artery disease. Clin Appl Thromb Hemost. 2014; 20:473-7.

To cite this article: Ahmed Elamragy, Ahmed Hussein, Ayman Kaddah, Marwa Meshaal Neutrophil: Lymphocyte Ratio Predicts Coronary Artery Disease Severity in Non-ST Elevation Myocardial Infarction Using Syntax Score. BMFJ 2021; 38(2): 465-473, DOI: 10.21608/bmfj.2021.52406.1353 